\title{
The efficacy of natalizumab in patients with relapsing multiple sclerosis: subgroup analyses of AFFIRM and SENTINEL
}

\author{
Michael Hutchinson - Ludwig Kappos - Peter A. Calabresi - Christian Confavreux · Gavin Giovannoni • \\ Steven L. Galetta - Eva Havrdova · Fred D. Lublin · David H. Miller · Paul W. O'Connor · \\ J. Theodore Phillips · Chris H. Polman · Ernst-Wilhelm Radue · Richard A. Rudick • \\ William H. Stuart · Andrzej Wajgt · Bianca Weinstock-Guttman · Daniel R. Wynn • \\ Frances Lynn · Michael A. Panzara $\cdot$ for the AFFIRM and SENTINEL Investigators
}

Published online: 29 May 2009

(C) Springer-Verlag 2009

Erratum to: J Neurol (2009) 256:405-415

DOI 10.1007/s00415-009-0093-1

Figs. 1a, 2a and 3a have been published incorrectly. The correct figures are reproduced here.

The online version of the original article can be found under doi:10.1007/s00415-009-0093-1.

M. Hutchinson $(\square)$

Dept. of Neurology, St. Vincent's University Hospital, Dublin, Ireland

e-mail: mhutchin@iol.ie

L. Kappos · E.-W. Radue

Dept. of Neurology, University Hospital Basel,

Basel, Switzerland

P. A. Calabresi

Dept. of Neurology,

The Johns Hopkins University School of Medicine,

Baltimore, MD, USA

C. Confavreux

Hôpital Neurologique, Lyon, France

G. Giovannoni - D. H. Miller

Institute of Neurology, London, UK

\section{S. L. Galetta}

Dept. of Neurology,

University of Pennsylvania School of Medicine,

Philadelphia, PA, USA

E. Havrdova

General Teaching Hospital, Prague, Czech Republic

F. D. Lublin

Mt. Sinai School of Medicine, New York, NY, USA
P. W. O'Connor

St. Michael's Hospital, Toronto, ON, Canada

J. T. Phillips

Multiple Sclerosis Center at Texas Neurology, Dallas, TX, USA

C. H. Polman

Vrije Universiteit Medical Center, Amsterdam, The Netherlands

R. A. Rudick

Mellen Center for Multiple Sclerosis Research,

Cleveland Clinic Foundation, Cleveland, OH, USA

W. H. Stuart

MS Center of Atlanta, Atlanta, GA, USA

A. Wajgt

Silesian Medical University, Katowice, Poland

B. Weinstock-Guttman

Baird Multiple Sclerosis Center,

State University of New York at Buffalo,

Buffalo, NY, USA

D. R. Wynn

Consultants in Neurology, Multiple Sclerosis Center,

Northbrook, IL, USA

F. Lynn · M. A. Panzara

Biogen Idec, Cambridge, MA, USA 
No. of patients (placebo, natalizumab)

\begin{tabular}{|c|c|c|}
\hline $\begin{array}{l}\text { No. of relapses } \\
\text { in the year } \\
\text { prior to screening }\end{array}$ & $\begin{array}{r}1 \\
2 \\
\geq 3\end{array}$ & $\begin{array}{l}(180,368) \\
(102,197) \\
(27,56)\end{array}$ \\
\hline $\begin{array}{l}\text { EDSS score at } \\
\text { baseline }\end{array}$ & $\begin{array}{l}\leq 3.5 \\
>3.5\end{array}$ & $\begin{array}{l}(278,548) \\
(37,79)\end{array}$ \\
\hline $\begin{array}{l}\text { Baseline no. } \\
\text { of T2 lesions }\end{array}$ & $\begin{array}{l}<9 \\
\geq 9\end{array}$ & $\begin{array}{l}(15,29) \\
(300,598)\end{array}$ \\
\hline $\begin{array}{l}\text { Baseline no. } \\
\text { of Gd+ lesions }\end{array}$ & $\begin{array}{r}0 \\
\geq 1\end{array}$ & $\begin{array}{l}(172,308) \\
(143,319)\end{array}$ \\
\hline Gender & $\begin{array}{r}\text { Female } \\
\text { Male }\end{array}$ & $\begin{array}{l}(211,449) \\
(104,178)\end{array}$ \\
\hline $\begin{array}{l}\text { Age at baseline } \\
\text { (years) }\end{array}$ & $\begin{array}{l}<40 \\
\geq 40\end{array}$ & $\begin{array}{l}(188,399) \\
(127,228)\end{array}$ \\
\hline
\end{tabular}

No. of patients (placebo, natalizumab)

Fig. 2a

Fig. 1a

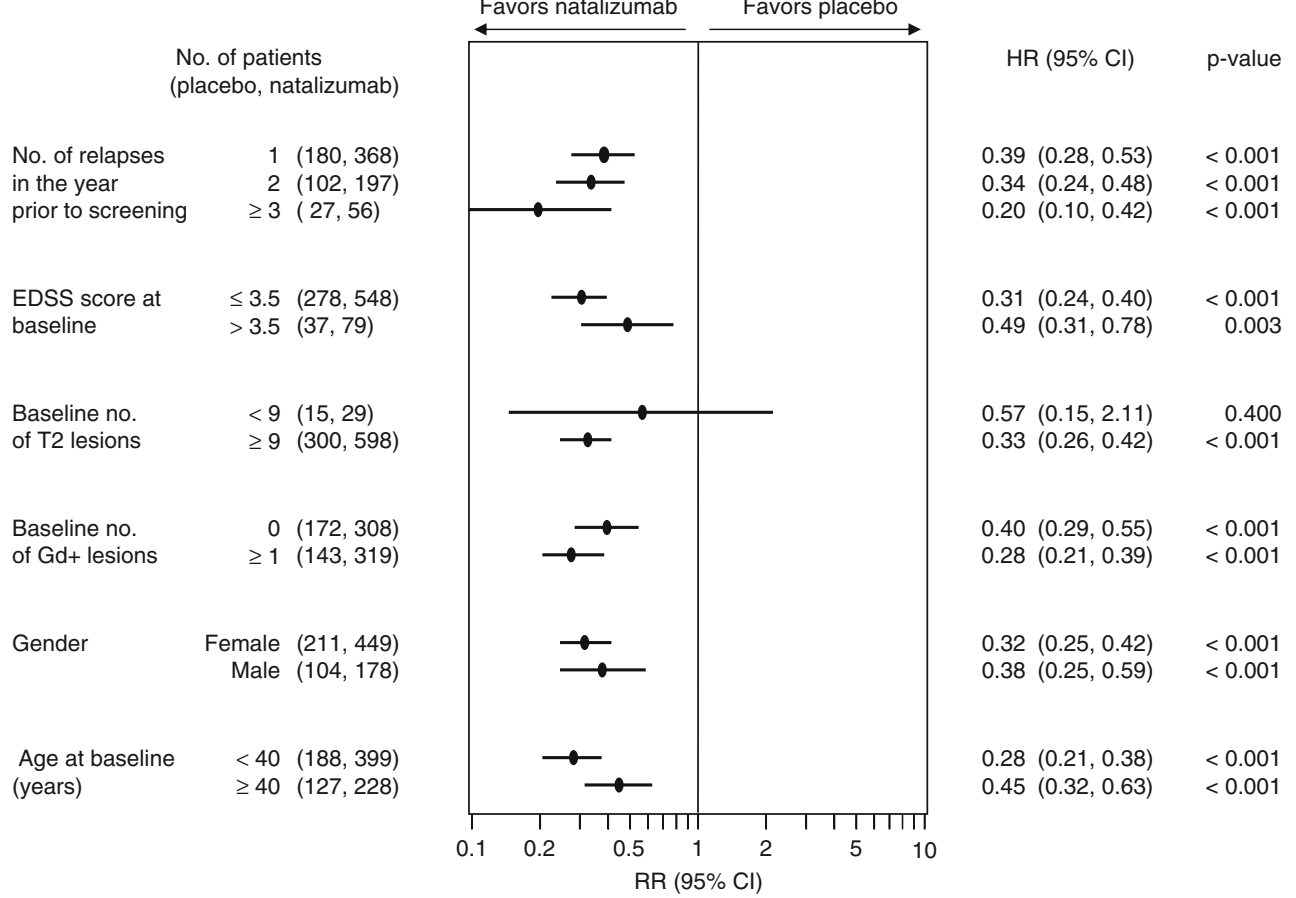

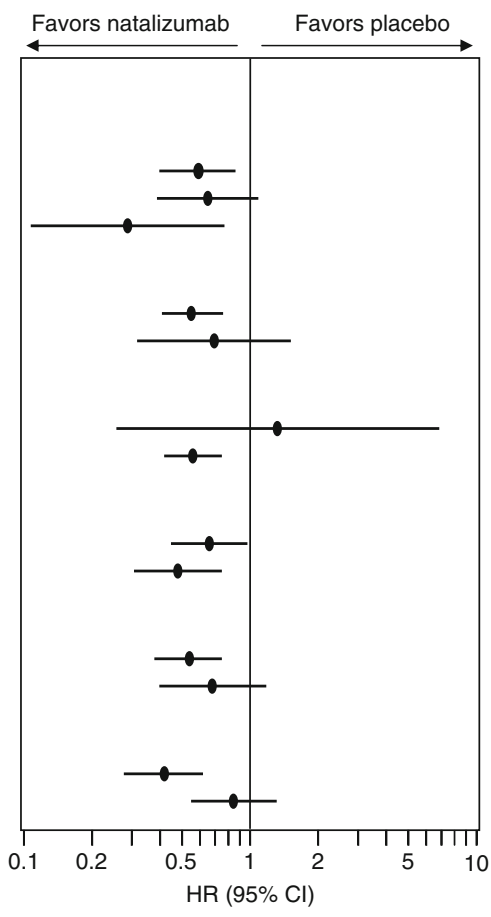




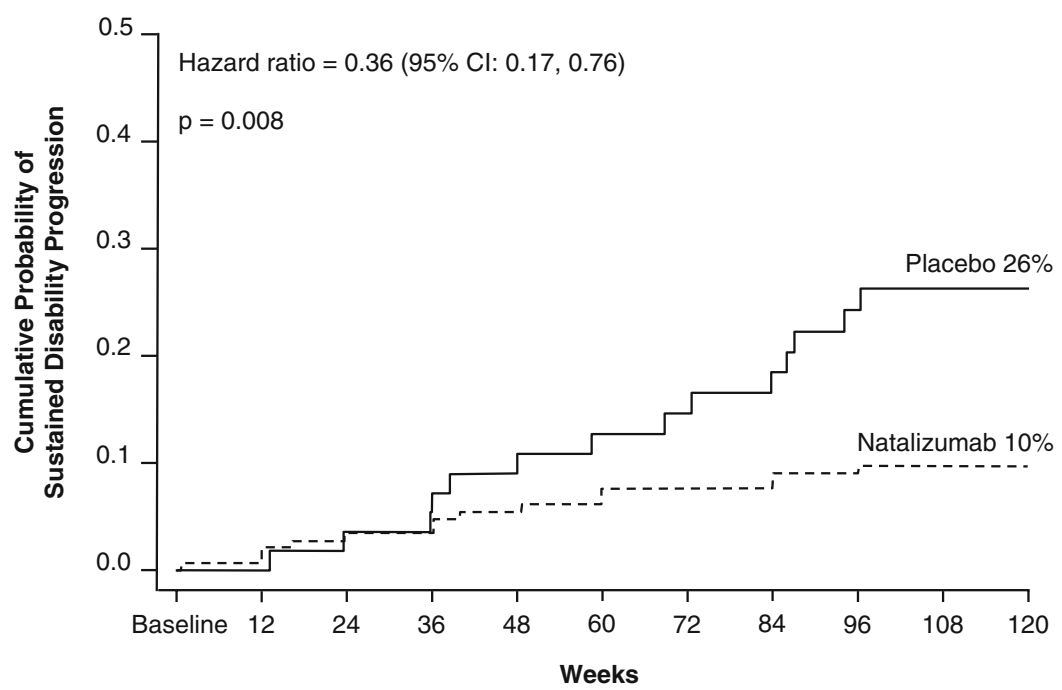

\begin{tabular}{rcccccccccc}
\multicolumn{11}{c}{ No. of patients at risk } \\
\hline Placebo & 61 & 57 & 54 & 51 & 47 & 46 & 45 & 42 & 39 & 36 \\
Natalizumab & 148 & 144 & 141 & 140 & 137 & 131 & 130 & 128 & 123 & 123
\end{tabular}

Fig. 3a 